\title{
An Improved Algorithm of Fuzzy on the QoS Evaluation of Web Services Based on OWL-QoS Ontology ${ }^{*}$
}

\author{
Zhang Cuixiao ${ }^{1}$, Zhang Guobing ${ }^{1}$, Xing Shuxia ${ }^{2}$ and $\mathrm{Xu} \mathrm{Li}^{1}$ \\ ${ }^{1}$ School of Information Science and Technology, Shijiazhuang Tiedao University, Hebei, China \\ ${ }^{2}$ Shijiazhuang Branch Huaxia Bank, Hebei, China \\ Zhangcx1996@126.com, zhanggb@stdu.edu.cn,xsx0213@163.com
}

\begin{abstract}
Presently, there is not a complete description and resolution method about the redundancy membership degree of Web service's computing properties among the methods of Web service quality evaluation. In this paper, Web service description is extended based on the OWL-QoS(Ontology Web Language- Quality of Service) Ontology, web service QoS evaluation model is established, the algorithm of fuzzy comprehensive evaluation is improved on differentiating redundancy membership degree. It eliminates redundant data in membership degrees of index which is useless for objective classification. It realizes membership degree conversion, and the conversion process will not add any new prior knowledge. The algorithm is used in the Web Service QoS evaluation. Finally, the practicality of this algorithm is verified by an instance.

Index Terms - Web Service, Quality of Service, Fuzzy Theory, Comprehensive Evaluation
\end{abstract}

\section{Introduction}

With the rapid growth of the number of Web services, users face the problem of how to find the optimal service from huge Web services. Service quality is the key to this question. It describes the ability to meet consumer's demand. The QoS (Quality of Service) reflects the qualitative and quantitative aspects of the Web service. Therefore, Web service quality evaluation becomes a major problem. If there is no a good solution, the development, selection, monitoring, charging and many problems of Web service will be influenced [1].

From the point of sample statistical calculating weight based on information entropy, Reference [2] gives an evaluation model. From the view of Web service quality and relative service quality evaluation, Reference [3] puts forward an algorithm of calculation Web service quality comprehensive evaluation value. Reference [4] gives a Web service QoS evaluation mechanism based on QoS ontology. Applying the fuzzy mathematics theory to describe the QoS attribute value, fuzzy comprehensive evaluation technique is applied to Web service QoS evaluation in [5]. However,these methods are all static and unilaterally modeling and evaluation for Web service.Such as they did not consider the influence of redundancy data on calculation of attribute value, not reflected the service quality indicators dynamic selection and user feedback information at various stages, etc.

QoS quantitative concept is introduced to Web service QoS evaluation model in this paper. Combined with QoS authentication center actively monitoring and user QoS feedback mechanism, this paper puts forward a fuzzy comprehensive evaluation algorithm of membership degree conversion. Finally, the practicality of this algorithm is verified by an instance.

\section{Web Service QoS Evaluation Model}

There are a lot of Web services with same function, QoS parameters become the most obvious difference among them. To realize the Web service quality evaluation, QoS model should be established and the main evaluation parameters should be determined first, and then choose appropriate algorithm of quality evaluation in service selection, monitoring, management and accounting process.

\section{A. Architecture of Model}

To show the dynamic multi-level characteristics of Web service QoS model, and the influence on the result of the quality evaluation from the customer's participative process, this paper proposes the customer's feedback factor as a service provider reference information guide the adjustment of the service.

In order to make the service quality comprehensive evaluation more profitably, the traditional Web service framework is extended, and a new QoS evaluation model based on ontology is put forward. Service information released by Service publishers and request information from service demander are described using OWL - QoS ontology, as well as the service matching. In addition, the matching engine is introduced into this model to complete function matching, QoS matching and quality evaluation.

As shown in Fig. 1, the service provider describes the service provided based on the OWL - QoS, including the function attributes and QoS information, and register information to a UDDI registration center through the OWLQoS/UDDI converter. Services requester (Service Consumer) describes the required service based on the OWL - QoS, including Service function attributes, QoS information and some limited attribute information according to their need. Service registration center (Service Registry) mainly process Service distribution and Service search request, after introducing ontology semantic description information, can provide Service OWL - QoS/UDDI converter more accurate to the service requester, because Service publishers and services requester provide information Service based on OWL - QoS ontology, so they need this converter to convert OWL ontology - QoS Service information to the registration center.

\footnotetext{
* This work is partially supported by Hebei science and technology projects \# 10213516D and natural science foundation of Hebei \#F2013210109
} 
Matching engine mainly completes service matching, including function matching, QoS information matching and service quality evaluation. According to the requested service function description, search services with the same function in UDDI, then according to the service requester's QoS information (the QoS information must be filtered by experts firstly, in order to ensure the minimum service performs conditional) demand or user preferences of QoS limited properties, filter the same function service found, make it satisfy the service requester, finally evaluate filtered service with the fuzzy algorithm.

\section{B. Web service QoS quality attribute}

Web service quality evaluation factors are mainly depended on the user's preferences. After domain expert opinion filtering, In view of practical and measurability consideration [6], this paper considers service QoS attribute mainly from the view of performance, usability, accessibility, integrity, reliability.

1) performance: Web service performance shows how soon a service request can be completed, it can be measured according to the throughput and delay and response time.

2) usability: usability shows the possibility of service available. Larger value shows service is available all the time, and the smaller value shows whether service is available in a certain time is unpredictable.

3)accessibility: it shows service level to Web service requested.

4) integrity: it refers to how to maintain the correctness of the initial condition for Web service, including data integrity and transaction integrity.

5) reliability: it shows the level of maintenance service and the service quality, in another sense, reliability is refers to the sending and receiving message of guarantee and orderly transfer (consistency) between the service requester and the service providers [7]. It includes fault resistance, consistency, and maintainability.

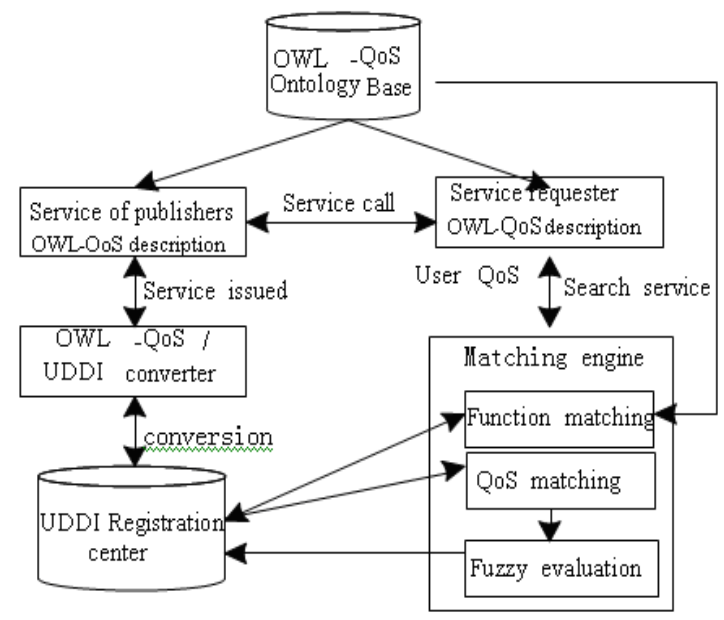

Fig. 1 QoS evaluation model based on ontology OWL - QoS

\section{Related Algorithm}

Assume that there are $m$ kinds of index influenced the target state $Q$, and each index is divided into $P$ levels, level $K$ is expressed by $C_{k}(k=1 \sim p)$, and level $C_{k}$ is better than level $C_{k+1}$. If the membership degree of index $\mathrm{j}$ of $Q$ belong to the level $C_{k}$ is known and expressed by $\mu_{j k}(Q)$,and meet the goal:

$$
0 \leq \mu_{j k}(Q) \leq 1, \sum_{k=1}^{p} \mu_{j k}(Q)=1,(k=1 \sim p, j=1 \sim m)
$$

Now, we need to calculate membership degree of $\mathrm{Q}$ belong to the level $C_{k}$ which expressed by $\mu_{j k}(Q)$.

\section{A. Distinguish Right}

Firstly, index membership degree and numerical value of membership degree which is eligible for the calculation of the target membership should be determined[8]. In membership degree $\mu_{j k}(Q)$, centralized or decentralized degree of $k$ can be described by entropy of membership degree $H_{j}(Q)$ [9], and so, the real number $\alpha_{j}(\mathrm{Q})$ can be represented as function of entropy:

$$
\begin{aligned}
& H_{j}(Q)=-\sum_{k=1}^{p} \mu_{j k}(Q) \cdot \log \mu_{j k}(Q) \\
& v_{j}(Q)=1-H_{j}(Q) / \log p \\
& \alpha_{j}(Q)=v_{j}(Q) / \sum_{t=1}^{m} v_{t}(Q) \quad(j=1 \sim m)
\end{aligned}
$$

So the real number $\alpha_{j}(\mathrm{Q})$ is called distinguish right of $\mathrm{j}$ to $\mathrm{Q}$, clearly distinguish right $\alpha_{j}(\mathrm{Q})$ meets:

$$
0 \leq \alpha_{j}(Q) \leq 1, \sum_{j=1}^{m} \alpha_{j}(Q)=1
$$

Distinguish right is a kind of filter, in membership conversion process, it can filter out the redundant indicators membership and the redundancy membership numerical which is no use on the target $Q$ classification.

\section{B. Comparableworth and membership conversion}

$\beta_{j}(Q)$ is the importance weights of $j$-th of $\mathrm{Q}$ to $\mathrm{Q}$ ( is calculate according the domain expert prior knowledge), then:

$$
\beta_{j}(Q) \cdot \alpha_{j}(Q) \cdot \mu_{j k}(Q) \quad(k=1 \sim p)
$$

the above formula is membership of comparable value, named: $k$ class ratio. Obviously, class ratio of different index has comparable and direct additivity. Therefore, the sum of the comparableworth of $k$ class of target $Q$ target is shown by (7). Obviously, relatively bigger value of $M_{k}(Q)$ shows the degree of $Q$ belonging to the class $C_{k}$ is bigger.

$$
M_{k}(Q)=\sum_{j=1}^{m} \beta_{j}(Q) \cdot \alpha_{j}(Q) \cdot \mu_{j k}(Q),(k=1 \sim p)
$$


Finally, we can get that membership $\mu \mathrm{k}(\mathrm{Q})$ of $Q$ belonging to the class $C_{k}$ is shown by type (8)

$$
\mu_{k}(Q) \stackrel{\Delta}{=} M_{k}(Q) / \sum_{t=1}^{p} M_{t}(Q),(k=1 \sim p)
$$

So far, membership conversion from index membership to target membership is realized, during conversion not increase any prior knowledge, nor cause classification information distortion [9]. The membership conversion algorithm can be summarized as "filter, ratio, synthesis". "filter" refers to filter with distinguish right, which filters out the target classification inoperative redundant indicators membership and redundancy numerical of index membership degree; "ratio" refers to calculate Comparableworth and generate total value; "synthetic" refers to calculate the target membership.

\section{Application Example}

Suppose there are multiple Web services of ordering ticket online, and their QoS attributes have more than one. Now we evaluate one of these services using the above membership degree conversion algorithm. Evaluation attributes are: accessibility, usability, performance, reliability and integrity. Service provider defines the specific values for each attribute QoS, specific QoS parameters as is shown in TABLE 1.

\section{A. Web Service QoS Attribute Evaluation Matrix}

In order to realize the above algorithm to evaluate the Web service, firstly, use fuzzy comprehensive evaluation to make decision for QoS [4]. Web service QoS fuzzy evaluation matrix is shown in TABLE 1:

TABLE 1 Web service QoS fuzzy evaluation matrix

\begin{tabular}{|c|c|c|}
\hline $\begin{array}{l}\text { Index } \\
\text { level one }\end{array}$ & $\begin{array}{l}\text { Index } \\
\text { level two }\end{array}$ & $\begin{array}{l}\text { Membership vector } \\
\text { (very satisfied. Satisfactory. } \\
\text { General. Not too satisfactory. } \\
\text { unsatisfied) }\end{array}$ \\
\hline \multirow{3}{*}{$\begin{array}{l}\text { Performance } \\
(0.233) \mathrm{A} 1\end{array}$} & throughput (0.272)B11 & $\left(\begin{array}{lllll}0.00 & 0.00 & 0.46 & 0.31 & 0.23\end{array}\right)$ \\
\hline & delay $(0.370) \mathrm{B} 12$ & $\left(\begin{array}{lllll}0.00 & 0.23 & 0.38 & 0.39 & 0.00\end{array}\right)$ \\
\hline & response time $(0.358) \mathrm{B} 13$ & $\left(\begin{array}{lllll}0.23 & 0.17 & 0.05 & 0.35 & 0.20\end{array}\right)$ \\
\hline $\begin{array}{l}\text { Usability } \\
(0.251) \mathrm{A} 2\end{array}$ & & $\left(\begin{array}{lllll}0.41 & 0.32 & 0.00 & 0.18 & 0.09\end{array}\right)$ \\
\hline $\begin{array}{l}\text { accessibility } \\
(0.184) \mathrm{A} 3\end{array}$ & & $\left(\begin{array}{lllll}0.15 & 0.25 & 0.35 & 0.25 & 0.00\end{array}\right)$ \\
\hline \multirow{2}{*}{$\begin{array}{l}\text { integrity } \\
(0.175) \mathrm{A} 4\end{array}$} & data integrity $(0.58) \mathrm{B} 41$ & $\left(\begin{array}{lllll}0.32 & 0.26 & 0.32 & 0.20 & 0.00\end{array}\right)$ \\
\hline & $\begin{array}{l}\text { transaction integrity } \\
(0.42) \text { B42 }\end{array}$ & $\left(\begin{array}{lllll}0.27 & 0.21 & 0.39 & 0.13 & 0.00\end{array}\right)$ \\
\hline \multirow{3}{*}{$\begin{array}{l}\text { reliability } \\
(0.157) \mathrm{A} 5\end{array}$} & fault resistance $(0.581) \mathrm{B} 51$ & $\left(\begin{array}{lllll}0.00 & 0.00 & 0.32 & 0.61 & 0.07\end{array}\right)$ \\
\hline & consistency (0.309)B52 & $\left(\begin{array}{lllll}0.00 & 0.25 & 0.50 & 0.25 & 0.00\end{array}\right)$ \\
\hline & maintainability $(0.110) \mathrm{B} 53$ & $\left(\begin{array}{lllll}0.00 & 0.25 & 0.50 & 0.25 & 0.00\end{array}\right)$ \\
\hline
\end{tabular}

\section{B. Calculation Steps Based on the Algorithm}

(1) as an example, calculation membership of A1, the calculation steps are as follows:

(1) evaluation matrix of A1 is follows:

$$
U\left(A_{1}\right)=\left(\begin{array}{lllll}
0.00 & 0.00 & 0.46 & 0.31 & 0.23 \\
0.00 & 0.23 & 0.38 & 0.39 & 0.00 \\
0.23 & 0.17 & 0.05 & 0.35 & 0.20
\end{array}\right)
$$

Using the $j$-th line of $U(A l)(j=1 \sim 3)$ to calculate distinguish right of the index $B_{1 j}$, archiving the distinguish weight vector:

$$
\alpha\left(A_{1}\right)=\left(\begin{array}{lll}
0.4553 & 0.4432 & 0.1015
\end{array}\right)
$$

(2)From TABLE 1, the importance weight vector of index $B_{11} 、 B_{12} 、 B_{13}$ :

$$
\beta\left(A_{1}\right)=\left(\begin{array}{lll}
0.272 & 0.370 & 0.358
\end{array}\right)
$$

(3) Calculation ratio of class $k$ of index $B_{1 j}$, the ratio matrix can be shown as follow:

$$
N\left(A_{1}\right)=\left(\begin{array}{ccccc}
0 & 0 & 0.05697 & 0.0384 & 0.0285 \\
0 & 0.0377 & 0.0623 & 0.06395 & 0 \\
0.0084 & 0.0062 & 0.0018 & 0.0126 & 0.0072
\end{array}\right)
$$

(4) We can calculate the comparable total value of the ratio matrix $A_{l}$ of class $k$ :

$$
M\left(A_{1}\right)=\left(\begin{array}{lllll}
0.0084 & 0.0439 & 0.1211 & 0.11495 & 0.0357
\end{array}\right)
$$

(5)we can calculate membership vector $U(A 1)$ by $M\left(A_{1}\right)$ :

$$
\mu\left(A_{1}\right)=\left(\begin{array}{lllll}
0.0259 & 0.1355 & 0.3737 & 0.3547 & 0.1102
\end{array}\right)
$$

In the similar way, we got $U(A 4), U(A 5)$ Web service QoS attribute fuzzy evaluation matrix $U(\mathrm{Q} o \mathrm{~S})$ is made up by the above matrixes. :

$$
U(\mathrm{QS})=\left(\begin{array}{ccccc}
0.0259 & 0.1355 & 0.3737 & 0.3547 & 0.1102 \\
0.41 & 0.32 & 0.00 & 0.18 & 0.09 \\
0.15 & 0.25 & 0.35 & 0.25 & 0.00 \\
0.2808 & 0.2236 & 0.3388 & 0.1568 & 0 \\
0 & 0.0879 & 0.3833 & 0.4834 & 0.0454
\end{array}\right)
$$

(2)According to same steps(1)for matrix $U(\mathrm{Q} o \mathrm{~S})$, we got web service QoS attribute membership degree vector shown as follow :

$$
\mu\left(Q_{0 S}\right)=\left(\begin{array}{lllll}
0.1759 & 0.2015 & 0.2675 & 0.2987 & 0.0564
\end{array}\right)
$$




\section{Calculation Comprehensive QoS Value}

The quantization value vector of evaluation grade \{very satisfied, quite satisfied, general, not too satisfied, unsatisfied $\}$ is shown as follow: $\left(n_{1}, n_{2}, n_{3}, n_{4}, n_{5}\right)=(5,4,3,2,1)$.

$$
\eta(\mathrm{Q} o \mathrm{~S})=\sum_{k=1}^{5} n_{k} \cdot \mu_{k}(q O s)
$$

is called as the composite value of Web service $Q o S$ attribute value : $\eta(q o s)=3.1418$

The evaluation of Web service $Q o S$ provides the basis to choose the right service for the requesters of Web services . The third institution can accurately calculate the value of Web service $Q o S$ provided by service publishers for user's selection; evaluation of the Web service $Q o S$ attribute not only can make the users of service get better service, but also can make providers of service constantly upgrade the service in order to improve the competitiveness on the Internet

\section{Conclusion}

Most of the existing fuzzy comprehensive evaluation algorithms are mainly based on "weighted sum", they have not solve the problem of redundant attribute value in the evaluation of Web service QoS. In this paper, from the view of target classification, a membership degree conversion algorithm is put forward, the algorithm can be called "filter, ratio, synthetic", It realizes conversion of membership degree without redundancy membership degree. Shown by algorithm, The evaluation conclusion is made by fuzzy evaluation matrix. Determining reasonably index membership degree vector and the importance of weight value of the sub-index is a basic computing aspect related to whether the evaluation conclusion is reliable.

In this paper, Web services is described using OWL-QoS ontology, matching service functional and non-functional properties, and then use the factors of quality membership degree conversion algorithm to evaluate web service. The evaluation result provides a comprehensive evaluation value of the Web service QoS. It provides the basis to level classification among Web services with same function, so that service requester can select the optimal Web services to meet their needs.

\section{References}

[1] Zhang Nan, Qiu Xuesong, "Fuzzy dimensional QoS model of the webbased service quality evaluation," Computer Engineering, vol.34, no.9, pp.42-44,2008.

[2] Zheng Xianghong, Li Yuanchun,and Zengzhi.(2007), "the Oriented Pragmatics Web service QoS Evaluation Model", Journal of University of Electronic Science and Technology, vol.36,no.6,pp.1478-1480,2007.

[3] Han Tao,Guo Qing, and Gao Ying, "Assessment algorithm based on a QoWS the Web service", Computer Science, vol.33,no.5,pp.60-62,2006.

[4] Gao Yachun,Zhang Qun, "the QoS ontology-based Web service description and selection mechanism", Computer Science, vol.35, no.12, pp.273-276, 2008.

[5] Wang Fei, Zou Shihong,and Shanzhi, "Web service QoS modeling based on fuzzy mathematics", Application Research of Computer, vol.24, no.4, pp.214-216, 2007.

[6] Sun Suyun,"UDDI extended web service QoS model."Computer Engineering,,vol.34, no.14, pp.132-134, 2008

[7] Gouscos, Kalikakis, and Georgiadis," An approach to modeling Web service QoS and provision price," In:Fourth Intl. Conf. on,pp.121-130, Dec.2003.

[8] M.Dong,R.Kothari," Look-ahead Based Fuzzy Decision Tree Induction," IEEE Transactions on Fuzzy Systems, vol.9,no.3,pp.461-468,2001.

[9] S.Mitra,K.M.Konwar, andS.K.Pal." Fuzzy Decision Tree, Linguistic Rules and Fuzzy Knowledge-based Network, Generation and Evaluation," IEEE Transactionson Systems,no.32, pp.328-339, 2002. 